\title{
HIGH-PERFORMANCE MULTI-BATCH FUEL MANAGEMENTS FOR THE ADVANCED SOLUBLE-BORON-FREE ATOM CORE
}

\author{
Xuan Ha Nguyen, Seongdong Jang, and Yonghee Kim* \\ Korea Advanced Institute of Science and Technology (KAIST) \\ 291 Daehak-ro, Yuseong-gu, Daejeon, 34141, Republic of Korea \\ xuanha@kaist.ac.kr, seongdong@kaist.ac.kr, yongheekim@kaist.ac.kr
}

\begin{abstract}
The autonomous transportable on-demand reactor module (ATOM), a 450 MWth PWR-type small modular reactor (SMR), is under development at Korea Advanced Institute of Science and Technology (KAIST). The ATOM core is designed for soluble-boron-free and passive autonomous load-following operations by utilizing successfully an advanced reactivity control technology, centrally-shielded burnable absorber (CSBA). To enhance the ATOM core safety, CrAl-coated Zircaloy-4 is adopted as an accident-tolerant-fuel cladding. For a long operational cycle, the reference ATOM core has primarily accomplished with a single-batch fuel management (FM). In this paper, for more flexible operation and enhanced fuel utilization, various multi-batch FMs are investigated while the core performance is maintained in terms of both neutronic and safety aspects. These aspects are refueling pattern, cycle length, burnup reactivity swing, discharge burnup, axial and radial power peaking factor (PPF), total PPF, and temperature coefficients. Several refueling types are examined: In-out (low leakage), out-in (flattened power), and randomly scattered schemes. In addition, new heavy reflector designs, $\mathrm{ZrO}_{2}$ and $\mathrm{PbO}$, are introduced instead of stainless steel reflector for an improved core performance. Moreover, a new CSBA loading pattern is also proposed for an effective reactivity control of multi-batch FM strategy. Numerical results show that with a two-batch FM the cycle length can achieve above 2 years with an average discharge burnup of 40 $\mathrm{GWd} / \mathrm{tU}$, while the burnup reactivity swing remains less than $1,200 \mathrm{pcm}$. On top of that, the coolant and fuel temperature coefficients are highly negative at the beginning of cycle and power profile is comparable to that with the single-batch FM. All calculations in these multiphysics assessments of the ATOM core are performed using a Monte Carlo-diffusion hybrid code system based on Monte Carlo Serpent 2 and nodal diffusion COREDAX codes.
\end{abstract}

KEYWORDS: ATOM, Soluble Boron Free, CSBA, Heavy Reflector, Multi-Batch Fuel Management

\section{INTRODUCTION}

PWR-type Small modular reactors (SMRs), one of Gen-IV reactor concepts, have gained significant international R\&D efforts recently. These reactors, based on most advanced and mature technology, are designed to be multi-purpose, compact, simple and less dependent on active control means. They are also promising in terms of economics, siting flexibility, and multiple modular construction. For enhanced inherent safety feature and further simplification, the soluble-boron-free (SBF) operation is favorable. It eliminates several disadvantages of the current commercial pressurized water reactors (PWRs) such us slow

\footnotetext{
${ }^{*}$ Corresponding author
} 
reactivity response, potentially positive moderator temperature coefficient at a high borated concentration, complicated chemical and control volume system, and boron-induced corrosion on structural materials.

A high-performance PWR-type SMR, named autonomous transportable on-demand reactor module (ATOM), is being recently developed at center for autonomous small modular reactor research (CASMRR). Along with design features of an integrated PWR, the 450 MWth ATOM core is designed for SBF and passive autonomous operations. Numerous studies have been done for the development and assessment of the ATOM. To achieve SBF operation, an advanced burnable absorber technology, centrally-shielded burnable absorber (CSBA), is utilized [1]. The core design, CSBA loading, and control rod pattern was investigated to achieve a long cycle of more than 3 years with discharge burnup of about $29.0 \mathrm{GWd} / \mathrm{tU}$ [2]. In addition, an accident-tolerant-fuel (ATF) cladding and new heavy reflector designs were proposed to improve the neutronic and safety performances of the core [3]. Meanwhile, the material and experimental investigations are currently underway for the CSBA-loaded fuel pellet and several preliminary results are presented at references [4][5][6]. Moreover, the feasibility of passive autonomous frequency and daily loadfollow operations were demonstrated using [7] [8]. Nevertheless, the ATOM core with single-batch FM results in relatively small discharge burnup, about $29 \mathrm{GWd} / \mathrm{tU}$ with SS-304 reflector and $31 \mathrm{GWd} / \mathrm{tU}$ with $\mathrm{PbO}$ reflector [3]. Therefore, a two-batch FM is used, in this study, to increase the utilization of the fuel and provide various operational schemes for practical application of the ATOM core.

In this paper, a two-batch FM with 36 fresh fuel assemblies (FAs) and 33 burned FAs is investigated in which several shuffling schemes such as in-out, out-in, and scattered schemes are used for the optimization of the FM. On the other hand, a new CSBA loading pattern is proposed to attain a small burnup reactivity swing (BRS) during the cycle for SBF operation and considerably low power peaking. In addition, both newly proposed radial heavy reflectors, $\mathrm{ZrO}_{2}$ and $\mathrm{PbO}$, are applied to enhance the cycle length and compared to the reference SS-304 one. The (n, 2n) reaction in $\mathrm{PbO}$ reflector is considered in the modeling by using reduced absorption cross section. In order to analyze the ATOM core performance, Monte Carlodiffusion two-step procedure is used [9]. Since there is no commercial physics code that can model accurately the complicated 3-D geometry of the CSBA-loaded FA, in this procedure the Monte Carlo Serpent 2 code is used to generate the homogenized group constants [10]. Whereas, the 3-D thermalhydraulics-coupled neutronic core calculation is performed by using a diffusion-based nodal code, COREDAX [11]. COREDAX code adapts a single channel analysis model which is assumed that the heat is only transferred in the radial direction and it ignores the coolant cross flow between channels. It should be noted that the two-step procedure is well validated against other nuclear regulator-approved codes [11].

\section{THE ATOM CORE DESIGN}

\subsection{Centrally-Shielded Burnable Absorber}

In LWRs, advanced burnable absorber (BA) designs have been widely employed to minimize the core excess reactivity effectively during normal operation along with the uses of control rod and soluble boron. However, current commercial BA technologies have several limitations that lower reactor performance such as degradation in fuel thermomechanical properties and limited location for control rod insertion [12]. On top of that, these BA designs are insufficient to achieve a BRS for an SBF operation. Hence, CSBA was proposed to pursue a high-performance SBF SMR [1]. The CSBA-loaded fuel pellet is a typical $\mathrm{UO}_{2}$ pellet with BA balls loaded in its centerline, as illustrated in Fig. 1. It can offer high neutronic flexibility by adjusting CSBA ball radius and the number of CSBA balls per pellet, which dictate the spatial self-shielding effect and its depletion rate. Among various BA materials, the best candidate for the CSBA concept is natural gadolinia, $\mathrm{Gd}_{2} \mathrm{O}_{3}$. Gadolinia is a well-proven and popular $\mathrm{BA}$ material with a negligibly small reactivity penalty and high melting temperature of $2420{ }^{\circ} \mathrm{C}$, and it is highly compatible with $\mathrm{UO}_{2}$ fuel under reactor operating condition. Furthermore, there is no concern regarding thermal conductivity degradation of the fuel due to presence of gadolinia since gadolinia ball is completely segregated from $\mathrm{UO}_{2}$ fuel [5] [6]. 
Hence, fuel enrichment can be maximized up to $5 \mathrm{wt} \%$. As the BA balls are loaded inside fuel pellet, CSBA is clearly classified into integral type. It is advantageous that BA resides within fuel rod even in abnormal scenarios. Nevertheless, total amount of fuel in the CSBA-loaded pellet is less than that in the typical one, because CSBA balls replace a certain amount of the fuel.

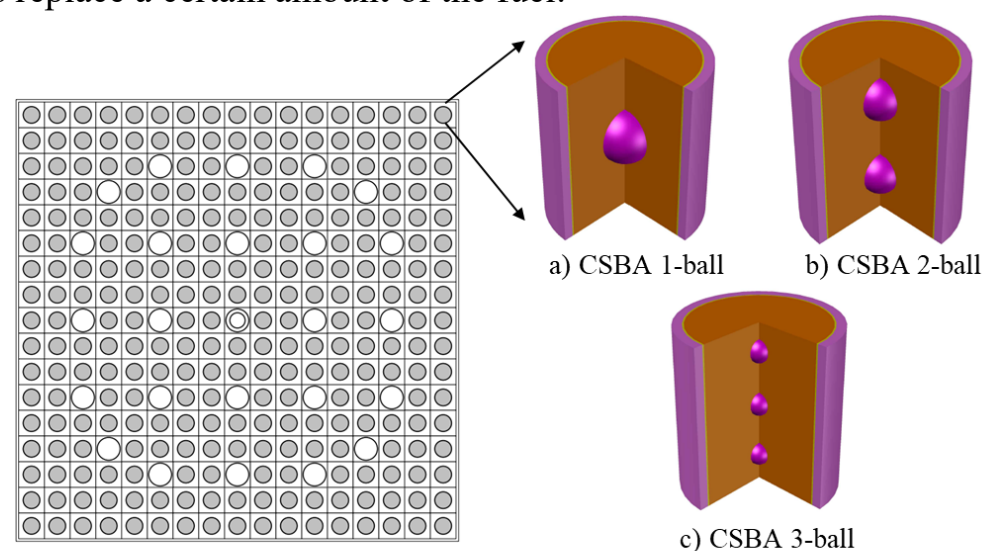

c) CSBA 3-ball

Figure 1: 17x17 CSBA-loaded Fuel Assembly

\subsection{The ATOM Core}

The major core parameters are presented in Table I, whereas the core layouts are depicted in Fig. 2 . The active core comprises of 69 17x17 FAs and each FA has 264 CSBA-loaded fuel rods, 25 guide tubes. There are three unique CSBA designs as depicted in Fig. 1. The fuel enrichment is $5.0 \mathrm{wt} \%$ with $95.5 \%$ theoretical density. At both ends of fuel rod, $5 \mathrm{~cm}$ fuel blankets with $2.0 \mathrm{wt} \% \mathrm{UO}_{2}$ are placed to minimize axial neutron leakage. In addition, the axial peaking factor and residual gadolinium at end of cycle can be dwindled by placing $5 \mathrm{~cm}$ BA cutbacks. In the original design, the stainless steel ,SS-304, was adopted as radial reflector [2]. On the other hand, SS-cladded $\mathrm{ZrO}_{2}$ and $\mathrm{PbO}$ reflectors are introduced to improve core cycle length further. Potential ATF claddings (surface-modified Zircaloy-4, iron-based cladding, etc.) were investigated with lattice calculation in terms of cycle length, discharge burnup, and required enrichment [13]. Cr15Alcoated Zircaloy-4 is selected as an ATF cladding for the ATOM core. The Cr15Al coating thickness is 0.03 $\mathrm{mm}$, while the Zircaloy- 4 outer radius is remained the same.

Table I: The Major Core Design Parameters

\begin{tabular}{|l|c|}
\hline \multicolumn{1}{|c|}{ Parameters } & Target Value \\
\hline Thermal power, power density & $450 \mathrm{MWth}, 26 \mathrm{~W} / \mathrm{gU}$ \\
\hline Active height, equivalent diameter & $200 \mathrm{~cm}, 201.6 \mathrm{~cm}$ \\
\hline Fuel materials, enrichment & $\mathrm{UO}_{2}, 5.0 \mathrm{wt} \%$ \\
\hline Radial reflectors & $\mathrm{SS}-304, \mathrm{ZrO}_{2}$, and $\mathrm{PbO}$ \\
\hline Inlet coolant tempt., coolant flow rate & $285^{\circ} \mathrm{C}, 2259 \mathrm{~kg} / \mathrm{s}$ \\
\hline CSBA design & $1-$ ball, 2-ball, and 3-ball \\
\hline BA material, density & $\mathrm{Gd}_{2} \mathrm{O}_{3}, 90 \%$ theoretical density \\
\hline FA type, number of FA & $17 \times 17,69$ \\
\hline ATF cladding design & $\mathrm{Cr} 15 \mathrm{Al}$-coated Zircaloy-4 \\
\hline Burnup reactivity swing & 1000 pcm \\
\hline
\end{tabular}




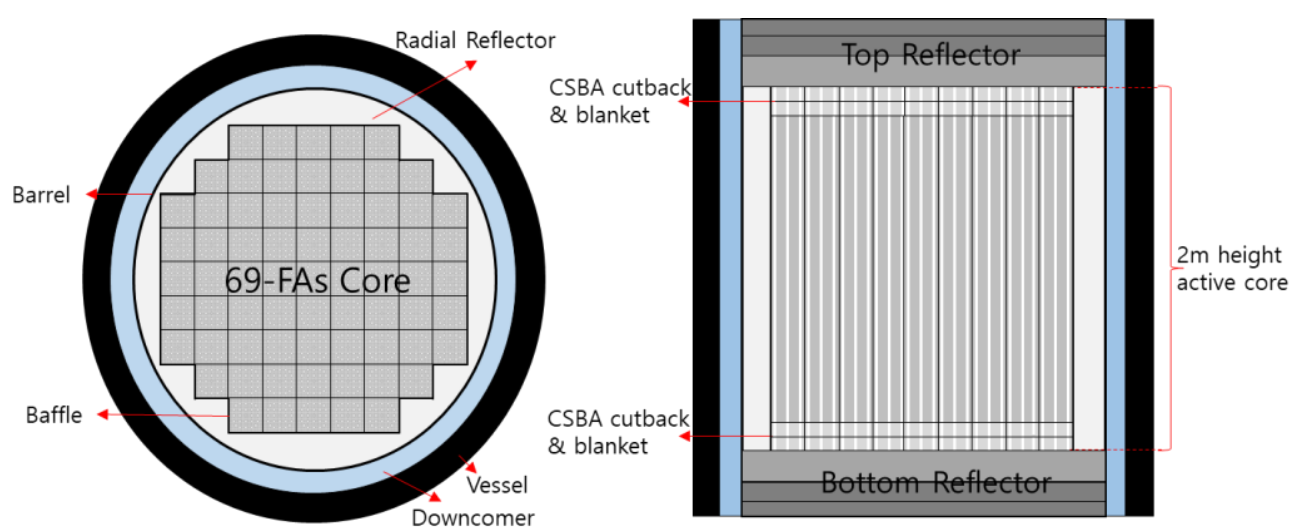

Figure 2: Radial and Axial Layouts of the ATOM Core

\section{TWO-BATCH FUEL MANAGEMENT FOR THE ATOM CORE}

\subsection{Selection of Fuel Shuffling Scheme}

The key idea of fuel shuffling optimization is to find the fuel pattern that enhances the utilization of the fuel while remaining other reactor design requirements such as low power peaking factor, discharge burnup within limitation, etc. In this paper, there are three typical shuffling schemes investigated. The first one is in-out scheme, a low leakage pattern, in which fresh FAs are placed in the inner regions and the burned FAs are placed in outer regions. Whereas, out-in scheme, a low power peaking pattern, inversely shuffles FAs compared to the first one. The random pattern can be seen in the third one, scattered scheme. The proposed patterns for the two-batch ATOM core is shown in Table II and FA numbering is shown in Fig. 3. 36 fresh FAs scheme is used, which is the minimum number of fresh FAs in a 69-FAs core, as we want to maximize average discharge burnup of the ATOM core. The E1 is fixed to be burned FA since use of fresh FA in the center must be avoided owing to high power peaking. For consistent comparison, the FA without BA is used in all of the fresh FA locations and the cycle burnup is set to be $22.0 \mathrm{GWd} / \mathrm{tU}$ for all of three schemes.

Table II: The Proposed Fuel Shuffling Schemes for the ATOM Core

\begin{tabular}{|c|c|c|c|c|c|c|}
\hline \multicolumn{2}{|c|}{ In-out } & \multicolumn{2}{|c|}{ Out-in } & \multicolumn{2}{|c|}{ Scattered } & \multirow{2}{*}{$\begin{array}{c}\text { No. of } \\
\text { FAs }\end{array}$} \\
\hline Fresh FA & Burned FA & Fresh FA & Burned FA & Fresh FA & Burned FA & \\
\hline E3 & E1 & E3 & E1 & E3 & E1 & 1 \\
\hline E3 & Discharged & E3 & Discharged & E3 & Discharged & 3 \\
\hline E2 & F3 & E5 & E4 & E2 & F3 & 4 \\
\hline E4 & E5 & F3 & E2 & E4 & E5 & 4 \\
\hline F2 & F3 & F3 & F2 & F2 & F3 & 4 \\
\hline F4 & F5 & F5 & F4 & $\mathrm{F} 4$ & G5 & 8 \\
\hline G3 & $\mathrm{H} 4$ & G5 & G4 & F5 & G4 & 4 \\
\hline G4 & G5 & H4 & G3 & H4 & G3 & 8 \\
\hline
\end{tabular}

The neutronic performances corresponding to the three schemes are compared in the Table III. It can be seen that with the use of in-out scheme the excess reactivities at beginning of equilibrium cycle (BOEC) and end of equilibrium cycle (EOEC) are the highest ones. It is due to neutron leakage is minimal with burned FAs loaded in the outermost locations. While out-in one presents a low BOEC reactivity as the fresh FAs at periphery locations maximize neutron leakage, hence, the EOEC reactivity is also smaller than that in in-out scheme. However, it advantageous that both the axial and radial peaking factors are minimal. On the other hand, the scattered scheme results in highest radial peaking, lowest EOEC reactivity, and considerable axial peaking and BOEC excess reactivity compared to other schemes. Since the ATOM core 
has a low power density and the target is to maximize discharge burnup, the in-out scheme is more favorable compared to the other ones. Hence, in the next section in-out scheme is used along with a newly-proposed CSBA loading for the two-batch ATOM core.

Table III: Neutronic Performances at Equilibrium Cycle for Several Shuffling Schemes

\begin{tabular}{|c|c|c|c|c|}
\hline Scheme & BOEC reactivity $(\mathrm{pcm})$ & EOEC reactivity $(\mathrm{pcm})$ & Radial peaking & Axial peaking \\
\hline In-out & 21959 & 942 & $1.955(\mathrm{E} 2)$ & 1.446 \\
\hline Out-in & 19431 & 739 & $1.488(\mathrm{E} 3)$ & 1.371 \\
\hline Scattered & 21469 & 680 & $2.013(\mathrm{E} 2)$ & 1.445 \\
\hline
\end{tabular}

\subsection{The CSBA Loading Scheme for the Two-batch ATOM core}

As aforementioned in the previous section, there are three unique designs of CSBA-loaded fuel pellet. The 1-ball design has the strongest self-shielding effect as the BA ball has the smallest surface area per unit volume, while 3-ball design has the lowest effect [2]. As the core with two-batch FM has smaller excess reactivity than that of the core with single-batch FM, the smallest self-shielded design, 3-ball, is favorable with the two-batch ATOM core. In general, 3-ball design with larger ball radius is loaded in the inner FAs where power is the highest and 3-ball designs with smaller ball radius are loaded in the outer FAs. After numerous trials and errors by adjusting the CSBA radii and the number of CSBA ball per pellet, the optimal BA loading for the ATOM core with SS-304 reflector is shown in Fig. 3 and Table IV. This BA loading scheme is also used for $\mathrm{ZrO}_{2}$ and $\mathrm{PbO}$ reflectors for a consistent comparison.

\begin{tabular}{|c|c|}
\hline Zone & FA type \\
\hline Zone A & Fresh FA \\
\hline Zone B & Fresh FA \\
\hline Zone C & Fresh FA \\
\hline & Burned FA \\
\hline
\end{tabular}

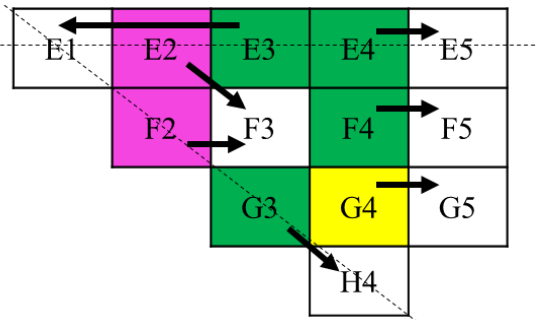

Figure 3: The CSBA Loading Scheme for the ATOM Core

Table IV: CSBA Designs Parameters

\begin{tabular}{|c|c|c|c|}
\hline Zone & Zone A & Zone B & Zone C \\
\hline CSBA Design & 3-ball, & 3-ball, & 3-ball, \\
CSBA ball radius & $\mathrm{r}=1.10 \mathrm{~mm}$ & $\mathrm{r}=1.00 \mathrm{~mm}$ & $\mathrm{r}=0.95 \mathrm{~mm}$ \\
\hline
\end{tabular}

Detailed neutronic performance of the two-batch ATOM core with SS-304 radial reflector is presented in both Fig. 4 and Table V. It can be seen that the core has a BRS of $1142 \mathrm{pcm}$, which is slightly larger than the target value. The cycle length of the core is about 803 days ( 2.2 years) corresponding to an average discharge burnup of $40.41 \mathrm{GWd} / \mathrm{tU}$. Meanwhile, the maximum FA discharge burnup, $51.84 \mathrm{GWd} / \mathrm{tU}$, clearly satisfies the upper limit burnup of $60 \mathrm{GWd} / \mathrm{tU}$. On the other hand, the neutronic performance of the cores with $\mathrm{ZrO}_{2}$ or $\mathrm{PbO}$ radial reflectors are also investigated and compared. Both $\mathrm{ZrO}_{2}$ and $\mathrm{PbO}$ reflectors improve significantly the cycle length as they have smaller neutron absorption cross section than SS-304 does [3], especially for $\mathrm{PbO}$ which is the heaviest material and induces (n, 2n) reaction. Nevertheless, the cores with $\mathrm{ZrO}_{2}$ or $\mathrm{PbO}$ reflectors accompany with higher BRS. The BRS is the maximum excess reactivity after xenon equilibrium. Since the CSBA scheme used here is for a consistent comparison and not yet optimized for cores with $\mathrm{ZrO}_{2}$ or PbO reflectors, further optimization of the CSBA is needed to achieve a comparable BRS with these reflectors. It is noted that the use of two-batch FM increases average discharge burnup significantly, above $40 \mathrm{GWd} / \mathrm{tU}$, while the average discharge burnup with single batch is only about 
$29 \mathrm{GWd} / \mathrm{tU}$ [2]. On the other hand, the rodded ATOM core is not evaluated here since the BRS is sufficient small and the control rod pattern can be similar to that of the single batch one [2].

Table V: Neutronic Comparison between Two-batch ATOM Cores with Various Reflectors

\begin{tabular}{|c|c|c|c|c|}
\hline Reflector & $\begin{array}{c}\text { BRS } \\
(\mathrm{pcm})\end{array}$ & $\begin{array}{c}\text { Average discharge } \\
\text { burnup }(\mathrm{GWd} / \mathrm{tU})\end{array}$ & $\begin{array}{c}\text { Maximum discharge } \\
\text { burnup }(\mathrm{GWd} / \mathrm{tU})\end{array}$ & $\begin{array}{c}\text { Cycle length } \\
(\text { day })\end{array}$ \\
\hline $\mathrm{SS}-304$ & 1142 & 40.41 & 51.84 & $803(+0)$ \\
\hline $\mathrm{ZrO} 2$ & 1383 & 41.11 & 51.73 & $823(+20)$ \\
\hline $\mathrm{PbO}$ & 1633 & 42.10 & 51.82 & $840(+37)$ \\
\hline
\end{tabular}

Figure 5 presents power profile of the core with SS-304 reflector at three burnup conditions. Thanks to CSBA, the radial power distribution is quite flat with the maximum peaking factor of about 1.43. On the other hand, the axial peaking, about 1.37, can be found at BOEC condition in which it shows a typical bottom-skewed shape due to high coolant density at bottom of the core. As burnup increases, the power shape becomes slightly top-skewed at EOEC condition as the fuel at the bottom half is highly burned. On the other hand, the axial temperature profiles are depicted in Fig. 6. Since single heat channel does not consider the axial heat transfer, the fuel temperature closely follows the power distribution as shown in Fig. 5. The maximum fuel temperature is $787.4 \mathrm{~K}$ at BOEC condition when axial power is peaked. Meanwhile, the coolant rise temperature from the bottom to the top is $35.5 \mathrm{~K}$.

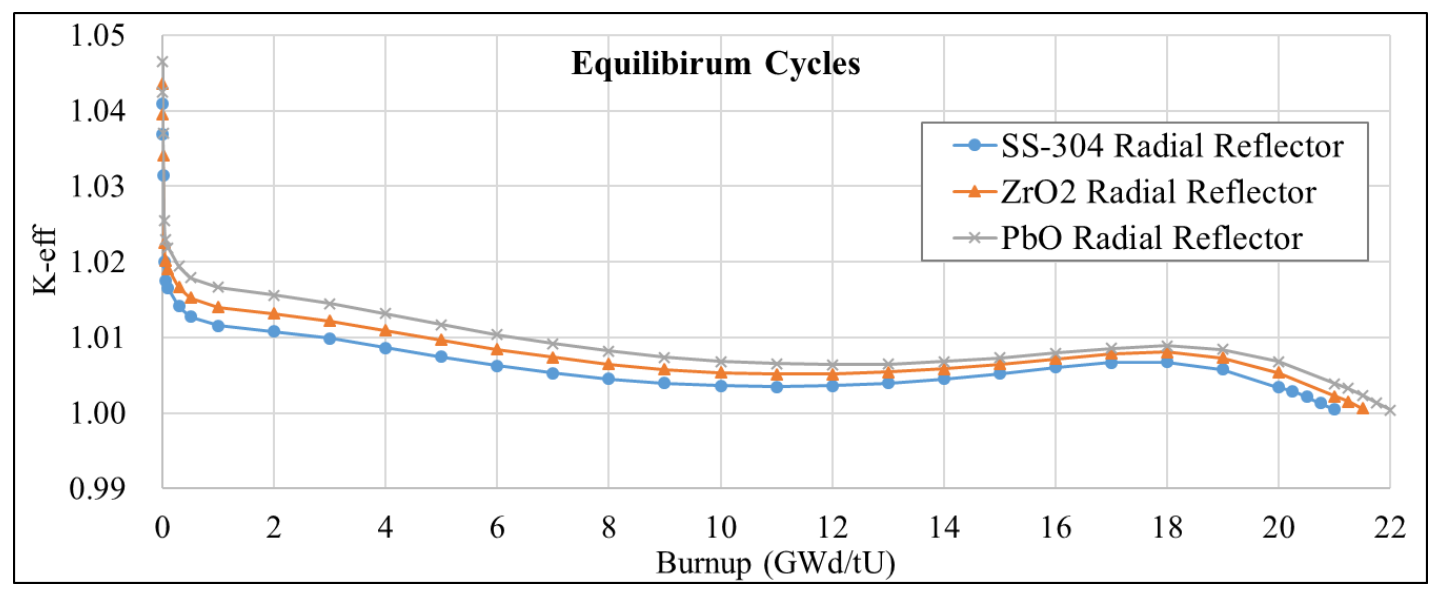

Figure 4: Multiplication Factor Evolution of the Two-batch ATOM Cores with Various Reflectors

\begin{tabular}{|l|l|l|l|l|}
\hline 1.120 & 1.127 & 1.249 & 1.162 & 0.796 \\
1.119 & 1.244 & 1.314 & 1.188 & 0.732 \\
1.141 & 1.429 & 1.372 & 1.226 & 0.657 \\
\hline & 1.146 & 1.178 & 1.142 & 0.770 \\
& 1.243 & 1.122 & 1.158 & 0.712 \\
& 1.388 & 1.055 & 1.186 & 0.641 \\
\cline { 3 - 5 } & & 1.213 & 1.058 & 0.633 \\
\multicolumn{2}{|l|}{} & 1.239 & 1.062 & 0.597 \\
BOEC & 1.260 & 1.080 & 0.542 \\
\hline MOEC & & 0.693 & 0.659 & \\
EOEC & & 0.614 & \\
\cline { 3 - 5 } & & &
\end{tabular}

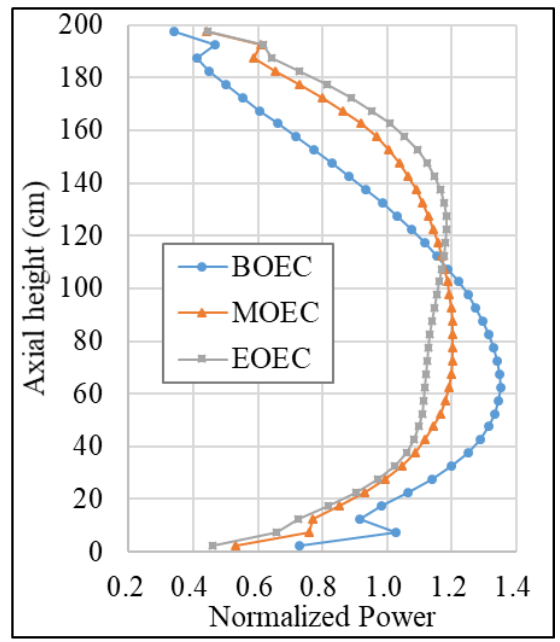

Figure 5: Radial and Axial Power Profile of The ATOM core with SS-304 Reflector 

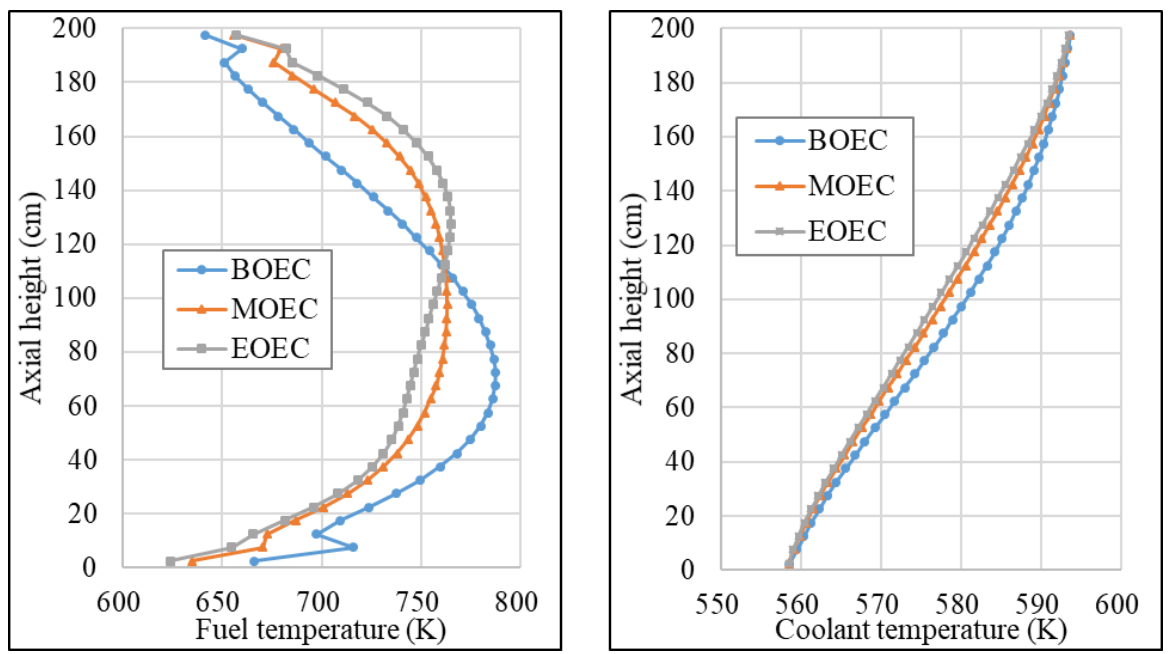

Figure 6: Axial Fuel and Coolant Temperature Distributions

Table VI lists temperature coefficients at BOEC and EOEC conditions. It is assumed that fuel temperature coefficient (FTC) and coolant temperature coefficient (CTC) are linear functions of temperature. It can be seen for the table that both FTC and CTC are highly negative at any condition due to absence of soluble boron. They becomes more negative at EOEC condition as results of neutron spectrum hardening and plutonium buildup, which enhance the inherent safety of the core. Moreover, it is demonstrated that the highly negative temperature coefficients are favorable for passive load-follow and frequency control operation [7]. It should be noted that FTC and CTC are evaluated by using Monte Carlo Serpent 2 only.

Table VI: The Fuel and Temperature Coefficients of the Two-batch ATOM Core

\begin{tabular}{|c|c|c|c|c|}
\hline Condition & $\begin{array}{c}\text { FTC } \\
(\mathrm{pcm} / \mathrm{K})\end{array}$ & $\begin{array}{c}\text { Standard deviation } \\
(\mathrm{pcm} / \mathrm{K})\end{array}$ & $\begin{array}{c}\mathrm{CTC} \\
(\mathrm{pcm} / \mathrm{K})\end{array}$ & $\begin{array}{c}\text { Standard deviation } \\
(\mathrm{pcm} / \mathrm{K})\end{array}$ \\
\hline BOEC & -2.47 & 0.21 & -47.32 & 1.67 \\
\hline EOEC & -3.29 & 0.19 & -62.44 & 1.50 \\
\hline
\end{tabular}

\section{CONCLUSIONS}

In this paper, a two-batch FM has been successfully developed by optimizing new CSBA loading strategy along with several fuel shuffling schemes. Consequently, the core can achieve a considerably small BRS with a cycle length more than two years. The discharge burnup of the fuel is significantly enhanced compared to the single-batch FM, while maintaining a flat radial power distribution throughout the cycle. In addition, new heavy reflectors, $\mathrm{ZrO}_{2}$ and $\mathrm{PbO}$, are investigated for an improved core performance. It is found that the use of $\mathrm{PbO}$ can extend the core cycle length about one month compared to that of SS-304. Hence, they are both promising reflector options along with SS-304 for a high-performance SMR and further FM optimization is needed for the cores with $\mathrm{ZrO} 2$ and $\mathrm{PbO}$ to minimize the BRS. On the other hand, the safety parameters, FTC and CTC, are also evaluated. The numerical results show that the twobatch ATOM core are inherently safe with highly negative temperature coefficients and highly favorable for the autonomous operation.

\section{ACKNOWLEDGMENTS}

This work was supported by the National Research Foundation of Korea (NRF) Grant funded by the Korean Government (MSIP) (NRF-2016R1A5A1013919). 


\section{REFERENCES}

[1] M. S. Yahya and Y. Kim, "A Centrally-Shielded Burnable Absorber for LWR fuel," in Proceedings of ICAPP 2017, Fukui and Kyoko, Japan, 2017.

[2] X. H. Nguyen, C. H. Kim and Y. H. Kim, "An advanced core design for a soluble-boron-free small modular reactor ATOM with centrally-shielded burnable absorber," Nuclear Engineering and Technology, vol. 51, pp. 369-376, 2019.

[3] X. H. Nguyen, S. Jang and Y. Kim, "Physics Study for Design Optimization of the Soluble-BoronFree Small Modular Reactor ATOM," in Global/ Topfuel 2019, Seattle, 2019.

[4] Q. M. Mistarihi, W. Park, K. Nam, M. S. Yahya, Y. Kim and H. J. Ryu, "Fabrication of oxide pellets containing lumped $\mathrm{Gd} 2 \mathrm{O} 3$ using $\mathrm{Y} 2 \mathrm{O} 3$-stabilized $\mathrm{ZrO} 2$ for burnable absorber fuel applications," Int' Journal of Energy Research, pp. 2141-2151, 2018.

[5] Q. Mistarihi, F. B. Sweidan and H. J. Ryu, "Thermo-physical properties of bulk Gd2O3 for fuel performance analysis of a lumped burnable absorber fuel design," in Transactions of the Korean Nuclear Society Autumn Meeting, October 25-27, Gyeongju, Korea, 2017.

[6] F. B. Sweidan and H. J. Ryu, "Composite Material Properties Simulation for The Fuel Performance Evaluation of Gadolinia-cored UO2 Fuel," in Transaction of the Korean Nuclear Society Autumn Meeting, October 25-27, Gyeongju, Korea, 2017.

[7] A. E. Abdelhameed, X. H. Nguyen and Y. Kim, "Feasibility of passive autonomous frequency control operation in a Soluble-Boron-Free small PWR," ANNALS OF NUCLEAR ENERGY, vol. 116, pp. 319-333, 2018.

[8] A. A. E. Abdelhameed, J. Lee and Y. Kim, "Physics conditions of passive autonomous frequency control operation in conventional large-size PWRs," Progress in Nuclear Energy, vol. 118, 2020.

[9] M. S. Yahya and Y. Kim, "A New Approach for a High-Performance Soluble-Boron-Free PWR core," PhD Thesis, KAIST, Daejeon, 2016.

[10] J. Leppänen, M. Pusa, T. Viitanen, V. Valtavirta and T. Kaltiaisenaho, "The Serpent Monte Carlo code: status, development and applications in 2013," Annals of Nuclear Engergy, vol. 82, pp. 142$150,2015$.

[11] B. Cho, S. Yuk, N. Z. Cho and Y. Kim, "User's manual for the rectangular three-dimensional diffusion nodal code COREDAX-2 version 1.8," KAIST, Daejeon, 2016.

[12] J. R. Secker and J. A. Brown, "Westinghouse PWR burnable absorber evolution and usage," in Transaction of American Nuclear Society, Orlando, 2010.

[13] X. H. Nguyen, S. D. Jang and Y. H. Kim, "Impacts of an ATF Cladding on Neutronic Performances of the ATOM SMR Core," in The 6th International Conference on Nuclear and Renewable Energy Resources (NURER2018), Jeju, Korea, 2018.

[14] Q. Mistahiri, M. S. Yahya and H. J. Ryu, "Fabrication Process of Neutron Absorber Inserted Oxide Fuel Pellet," in 12th Pacific Rim Conference on Ceramic and Glass Technology (PACRIM 12), including Glass \& Optical Materials Division Meeting (GOMD 2017), May 21-26th, Hawaii, USA, 2017. 